\title{
Fast-Track Surgery - Conditions and Challenges in Postsurgical Treatment: A Review of Elements of Translational Research in Enhanced Recovery after Surgery
}

\author{
Henry Hoffmann Christoph Kettelhack
}

Department of Surgery, University Hospital Basel, Basel, Switzerland

\section{Key Words}

Enhanced recovery after surgery $\cdot$ Fast-track surgery • Translational research

\begin{abstract}
Background: Enhanced recovery after surgery (ERAS) or fast-track surgery is a perioperative and postoperative care concept initiated in the early 1990s aiming to reduce the length of hospital stays following elective abdominal surgery. Twenty treatment items defined in the Consensus Guidelines established in 2009 were included in this concept. The success of ERAS depends highly on multidisciplinary teamwork and patient compliance. Several ERAS items and their impact on perioperative and postoperative care have recently been discussed. In this connection, translational research topics triggered increasing interest in ERAS and new impulses aimed at improving the ERAS concept. We thus reviewed the surgical literature to highlight the role of translational research items in ERAS. Methods: A literature search of Medline ${ }^{\circledR}$, PubMed $^{\circledR}$ and the Cochrane Database was performed. Two investigators independently reviewed the abstracts and appropriate articles were included in this review. Results: Articles have been selected. The advantages of the ERAS concept over conventional postoperative care were established by four meta-analyses and several reviews. But, due to the lack of standardization of the protocols, the
\end{abstract}

level of evidence is still low. The implementation of ERAS into clinical practice is furthermore hampered by the poor compliance with ERAS protocols and remains a challenge for the future. Moreover, recent trials challenge the role of some ERAS items, e.g. epidural anesthesia. Translational research trials investigating stress, immune and inflammatory response after surgery, new analgesic concepts, goal-directed fluid therapy and new drugs and substances to improve the outcome of ERAS provide first promising data but still need to be integrated in the ERAS concept. Conclusion: The Consensus Guidelines for ERAS are subject to the constant evolution of treatment strategies and implementation of translational research findings. Improvement of the compliance with ERAS protocols in surgical clinics and updating of ERAS items taking into account recent findings in translational research may improve the outcomes of ERAS but remain a long-term challenge in surgery for the next years.

Copyright $\odot 2012$ S. Karger AG, Basel

\section{Background}

The concept of 'fast-track surgery' or 'enhanced recovery after surgery' (ERAS) initiated by Kehlet [1] in the 1990s has been described in numerous studies in the last decade. The main goal of this concept is a reduction in the postoperative length of hospital stay (LOS). This is

\section{KARGER \\ Fax +4161306 1234 \\ E-Mail karger@karger.ch}

www.karger.com (c) 2012 S. Karger AG, Basel

0014-312X/12/0491-0024\$38.00/0

Accessible online at:

www.karger.com/esr
Henry Hoffmann, MD

University Hospital Basel, Department of Surgery

Spitalstrasse 21

$\mathrm{CH}-4031$ Basel (Switzerland)

E-Mail hoffmannh@uhbs.ch 
achieved by improved postoperative recovery using a multidisciplinary team approach maintaining cardiovascular, pulmonary, gastrointestinal, neurological and humoral functions. Following the first publications reporting a reduction in LOS to 2-3 days [2] and the implementation of Consensus Guidelines for ERAS [3], randomized studies revealed promising results favoring ERAS over conventional postoperative care [4]. The superiority of the ERAS concept has been further underlined by metaanalyses $[5,6]$. Lately, investigators increasingly aimed to study the impact of individual ERAS items (table 1) on the overall postsurgical outcome. These items have been investigated and discussed and led to a continuous evolution and variation of the original ERAS concept. Furthermore, improvement of the collaboration among involved treatment groups (e.g. general practitioners, nurses, anesthetists, pain teams, social care workers, outpatient departments and rehabilitation institutions) is one of the major challenges in ERAS.

After studying the impact of individual items on the overall outcome of the ERAS concept and their translation into clinical practice, new items and inputs from translational research drew the attention of scientists. Among these, humoral, metabolic, inflammatory and immune response after surgery, change in analgesic modalities and medical treatments, the role of tissue oxygenation and microcirculation, and goal-directed fluid therapy (GDT) were the main topics in translational research.

We reviewed the surgical literature on the ERAS items with a particular focus on translational research, regarding their impact and possible implementation into the ERAS concept.

\section{Methods}

Search Criteria and Data Extraction

We performed a review of the literature on ERAS, fast-track surgery and translational research identified by using Medline ${ }^{\circledR}$ and PubMed ${ }^{\circledR}$ and the Cochrane databases to give an overview regarding clinical studies, basic research findings and future challenges. The following key words were: 'enhanced recovery after surgery', 'ERAS', 'fast track surgery', 'postoperative ileus', 'length of hospital stay', 'laparoscopy', 'mast cells', 'gastrointestinal motility', 'immune response in surgery', 'inflammation response in surgery', 'tissue oxygenation', 'analgesic concept' and 'goal directed fluid management'.

With respect to the low evidence level in several translational research topics, original articles, clinical trials (phases I-III), comparative studies, results of consensus conferences, controlled clinical trials, in vitro studies, clinical studies, scientific reviews, meta-analyses and randomized controlled trials were eligible for this study. Regarding ERAS, only higher-evidence publications,

Fast-Track Surgery - Conditions and Challenges in Postsurgical Treatment e.g. meta-analyses, randomized controlled trials (RCTs) and reviews have been included in this study. The authors preliminarily reviewed abstracts, and studies not meeting the inclusion criteria were excluded.

\section{Results}

\section{The ERAS Concept}

Although recent meta-analyses $[5,6]$ proved that ERAS has a better outcome compared with conventional postoperative care, there is still a lack of standardization in these studies [7], which hinders achievement of higher levels of evidence. Routine use of ERAS protocol is safe [8], but compliance with ERAS protocols is only slowly improving in surgical clinics [9]. The main problem is the multidisciplinary integration of ERAS into routine clinical care, challenging surgeons, anesthetists, nurses, general practitioners and other involved health care professionals $[10,11]$.

Following numerous clinical studies, Lassen et al. [3] summarized 20 treatment items as Consensus Guidelines, which need to be considered when ERAS is used in clinical practice. Fifteen out of 20 items reached evidence grade A in ERAS (table 1).

\section{Implementation of ERAS into Clinical Practice}

Although compliance with ERAS protocol is increasing there is still a lack of standardization of using ERAS modalities [6, 12]. Several publications mention different ERAS items that have been included into their protocols, highlighting the ongoing evolution of ERAS protocols.

Ahmed et al. [7] have performed an interesting systematic review of the compliance with ERAS items. They identified 19 ERAS modalities (table 2) partially differing from the Consensus Guidelines (20 items) and included 11 clinical trials. They showed that none of the trials included all 19 ERAS items in their protocols. In fact, they identified a maximum of 14 items included by Ramirez et al. [8], 13 modalities included by Nygren et al. [13] whereas Schwenk et al. [14] and Kahokehr et al. [15] considered only 4 items. Ahmed et al. [7] reported that both clinical implementation (items not reported or included in the ERAS protocol) and compliance (in percent) showed widespread variation among the clinics (table 2), highlighting the necessity of further standardization. This improved the evidence in ERAS protocols, as shown in a recent Cochrane meta-analysis including only 4 RCTs [6]. Although a high compliance with the ERAS protocol is clearly correlated with improved outcome after surgery 
Table 1. ERAS items according to the Consensus Guidelines and health professional responsibility (adapted from Lassen et al. [3])

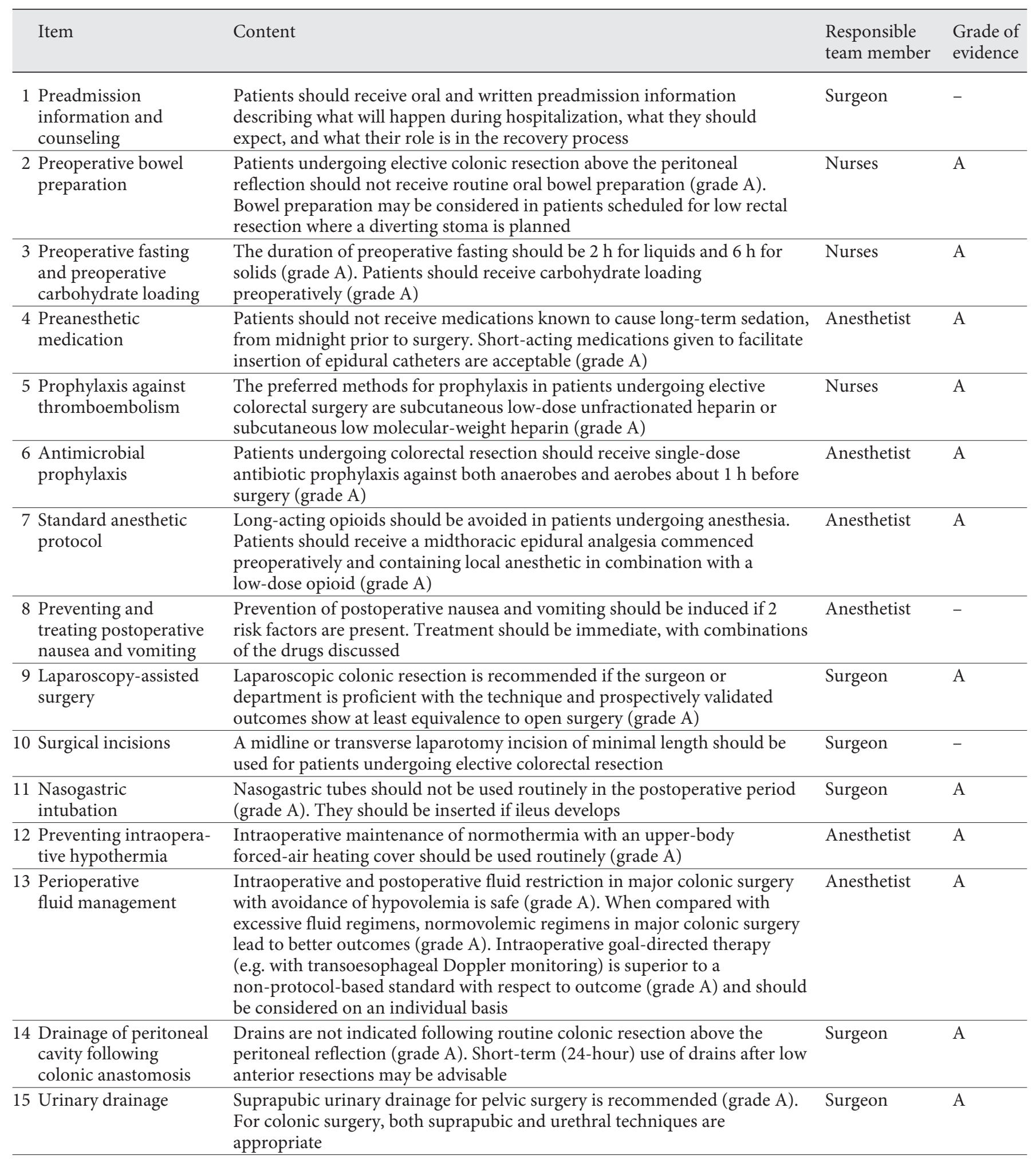


Table 1 (continued)

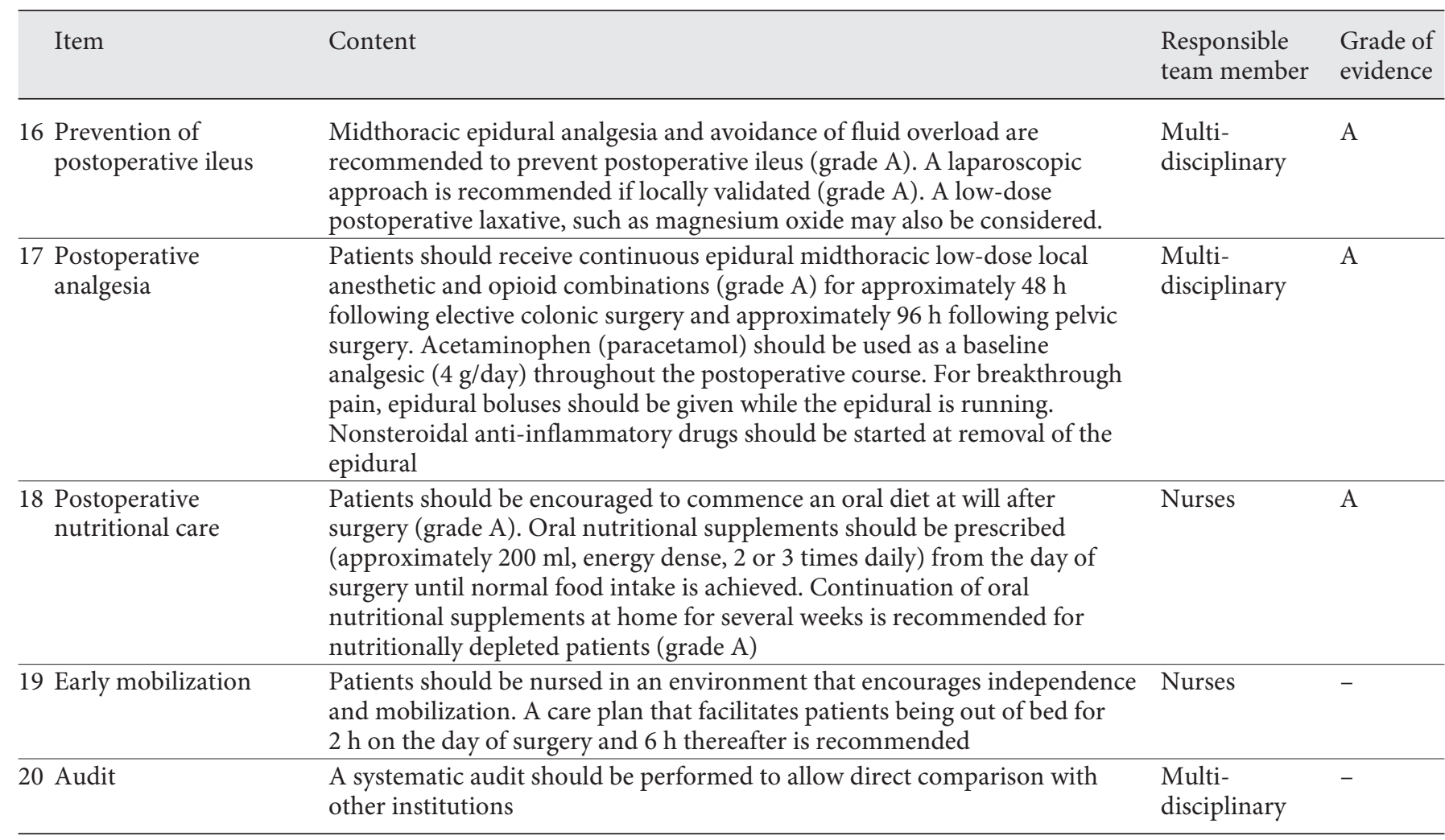

[7], pure facts do not seem to be convincing. A recent opinion survey of 1,390 surgeons in Germany and Austria revealed that implementation in routine surgical practice is not widely practiced [9]. Therefore, the challenge consists in a modification of traditional treatment strategies, overcoming interdisciplinary borders, accepting new ideas and coordination of the ERAS modalities in a prescheduled work flow by the responsible health professional (table 1).

\section{Impact of the ERAS Protocol on Surgical Outcome Parameters \\ Postoperative Ileus in ERAS}

The development of postoperative ileus (POI) as one of the major adverse events following abdominal surgery is based on the disturbance of the 'migration motor complex' [16]. POI has been characterized as an independent predicting factor for a prolonged LOS [17] and as the major factor accounting for in hospital morbidity regardless the type of operation [18]. Laparoscopy as part of the ERAS concept with limited surgical trauma has been shown to reduce POI significantly [19]. As shown in RCTs, routine bowel preparation for abdominal surgery had no effect on POI [20]. Several inter- and intracellular mechanisms and their role in POI are the focus of translational research (see below).

\section{LOS in ERAS}

Several studies reported a significant reduction in LOS when ERAS was compared with conventional postoperative care $[21,22]$ without increased readmission rates [21, 23]. A direct relationship between compliance with the ERAS protocol and a reduction in LOS was shown in further studies [7]. Vlug et al. [24] identified predictors for reduced LOS in ERAS, such as early, enforced mobilization, female gender, laparoscopic surgery and normal oral diet. Complications like POI [17] and increased postoperative pain [25] are predictors for extended LOS. Furthermore, nonmedical reasons like outpatient resources or limited social care facilities challenge LOS [10]. The use of laparoscopy significantly reduced LOS [18] and is therefore an implemented part of ERAS. In contrast, the 
use of epidural analgesia, one of the items in the Consensus Guidelines [3], has no effect on LOS and mobilization $[26,27]$ and hence should be critically investigated as to its impact on LOS and ERAS.

\section{Postoperative Complications in ERAS}

A correlation between ERAS and postoperative complications has been discussed. While randomized studies found decreased postoperative complications using ERAS [21,23], other publications showed at least comparable complication rates in ERAS and conventional postoperative care [22], challenging a clear advantage for ERAS protocols. Regarding the surgical approach, some authors reported reduced complications using minimally invasive techniques in RCTs [28]; another case-control publication failed to support this tendency [29]. The longstanding doctrine of routine use of nasogastric tubes and delayed oral feeding in major abdominal surgery in order to prevent complications has been disproved by a recent Cochrane analysis [30].

\section{Laparoscopy in ERAS}

Although laparoscopy plays an important role in elective and emergency abdominal surgery, and is widely used as a standard procedure in abdominal surgery, the value of laparoscopy in the ERAS concept has been investigated in clinical studies. Whereas ERAS has been shown to be beneficial in open surgery [22], several RCTs support the superiority of laparoscopy in ERAS [4, 28]. A recent 4-arm-trial comparing laparoscopic with open surgery and ERAS with standard postoperative care showed that laparoscopy combined with fast-track care (ERAS) leads to faster recovery of gastrointestinal motility and improves clinical recovery [31]. Although the operation costs and the rate of reoperations in laparoscopy are higher compared with conventional open surgery in ERAS [21], laparoscopy reduces complications, POI, LOS and the rate of admissions to intensive care units $[19,21,31]$ and, therefore, legitimately becomes a key item in ERAS.

\section{Analgesic Concepts in ERAS}

The postoperative pain concept in ERAS is part of an ongoing debate and still needs to be defined. Increased implication of the anesthetist and the development of dynamic analgesic regimens seem to be mandatory in further ERAS practice [32, 33].

A literature review regarding the impact of epidural anesthesia as one of the initial analgesic items in ERAS [3] provides different results regarding its effectiveness and role within the ERAS concept. A Cochrane analysis
Table 2. Implementation of (N/A) and adherence to ERAS items in clinical practice (adapted from a systematic review by Ahmed et al. [7] 2009)

\begin{tabular}{lcc}
\hline ERAS item & N/A & $\begin{array}{c}\text { Adherence } \\
\text { to ERAS items of } \\
\text { reporting studies, \% }\end{array}$ \\
& & \\
\hline Preoperatively & & $87-100$ \\
Counseling and education & $5 / 11$ & $0-100$ \\
No bowel preparation & $4 / 11$ & $61-100$ \\
Carbohydrate loading & $4 / 11$ & $0-60$ \\
No premedication & $7 / 11$ & 61 \\
Curtailed fasting & $10 / 11$ & \\
\hline Intraoperatively & & $79-100$ \\
Active warming & $7 / 11$ & 95 \\
High inspired O $(80 \%)$ & $10 / 11$ & $8-100$ \\
Epidural analgesia & $0 / 11$ & $25-96$ \\
Transverse incision & $8 / 11$ & $0-95$ \\
No use of NG tubes & $5 / 11$ & $46-81$ \\
Restricted i.v. fluids & $8 / 11$ & $0-47$ \\
No use of drains & $8 / 11$ & \\
\hline Postoperatively & & $5-100$ \\
Early oral fluids & $4 / 11$ & $5-100$ \\
Early oral food & $2 / 11$ & $70-100$ \\
Early mobilization & $1 / 11$ & $67-78$ \\
Oral analgesia & $7 / 11$ & \\
Avoidance of opiates & $9 / 11$ & \\
Postoperative laxatives & $10 / 11$ & \\
Routine antiemetics & $9 / 11$ & \\
\hline & & \\
\hline
\end{tabular}

[34] and a further meta-analysis [26] comparing intravenous opioid analgesia with midthoracic epidural analgesia demonstrated the beneficial effect of epidural analgesia in preventing postoperative ileus. In contrast, its role in ERAS is diminished due to the fact that epidural anestheticsmay cause hypotension and splanchnic hypoperfusion with an increased risk of cardiac events and anastomotic leaks [35]. Comparing epidural anesthetics with spinal anesthetics and patient controlled-anesthesia with opioids, spinal anesthetics and patient controlled-anesthesia were superior to epidural anesthetics regarding postoperative outcome [36]. Several studies demonstrated a positive effect of epidural anesthesia on outcome in open abdominal surgery $[37,38]$. But its role in laparoscopic surgery is diminished by the fact that it was not correlated [36]. Additional studies demonstrated no reduction in LOS and earlier oral food intake by using epidural anesthetics [27].

Interestingly, there is a consensus on the fact that extensive intravenous opioid administration should be 
avoided in ERAS; indeed, due to side effects and an increased rate of POI and LOS they should only be given to selected patients [39]. But this recommendation is challenged by the findings of Levy et al. [36, 40], who favor PCA using opioids or spinal anesthesia over epidural anesthesia in a systematic review and a clinical study. The role of COX-II inhibitors is well known: they mainly help reduce opioid consumption, and therefore should be part of multimodal pain management in ERAS [41].

Those results mitigate the role of epidural anesthesia in a laparoscopic surgery setting in ERAS, and protocols need to be reviewed and modified. Furthermore, the impact of PCA as a standard analgesic concept in ERAS needs to be reevaluated to clarify the controversial findings in the literature.

\section{Translational Research in ERAS}

After obtaining evidence using the ERAS concept in abdominal surgery $[5,6,42,43]$ and considering the controversial results when investigating the impact of separate ERAS items on outcome, several scientists lately concentrate on translational research. There are still unanswered questions, especially concerning the humoral, metabolic, inflammatory and immune response after surgery, the change in analgesic modalities, new pharmacological approaches, the role of tissue oxygenation and microcirculation, and the impact of GDT in perioperative care.

Inflammatory, Humoral and Immune Response after Surgery

Inflammation is the physiological reaction of vascularized tissue on manipulation, irritation and infection. This process is almost unavoidable in abdominal surgery with manipulation of the gut, where only a thin epithelial layer separates the microbial flora and the mucosal immune system.

Considering, that POI has been identified as one of the major factors for prolonged LOS and increased complications, the relationship between tissue inflammation and POI based on the concept of the 'motor migration complex' has recently been investigated. Neuronal innervation of the intestinal tract intensely communicates with the human immune system [44]. The complex interactions of mediators, e.g. interleukins (ILs) and tumor necrosis factor (TNF), induce invasion and degranulation of mast cells in the gut and subsequently lead to POI [45, 46]. Therefore, mast cell inhibition as a bona fide drug target could be one of the future treatment concepts to prevent POI [45]. In an experimental study, electric stim- ulation of the vagus nerve and central activation of anti-inflammatory cholinergic pathways by intravenous semapimod (an inhibitor of p38 mitogen-activated protein kinase) reduced tissue inflammation and the appearance of POI [47].

A recent RCT including 597 patients [22] investigated the impact of ERAS on surgical stress response and thus the degree of inflammatory activation. Measuring the nutrition index, the metabolism index (hemoglobin, albumin, prealbumin, triglycerides, transferrin, and nitrogen balance) and the stress index (cortisol, TNF- $\alpha$, IL-1 $\beta$, IL- 6 , and IFN- $\gamma$ ), the cascade of IL-1 $\beta$ inducing polymorphonuclear cells to migrate to the microvascular system and stimulate macrophages to produce IL- 6 and TNF- $\alpha$ was highlighted. Authors found a significant reduction in cortisol levels, IFN- $\gamma$ and TNF- $\alpha$ levels in the ERAS group after surgery compared with the control group whereas IL-1 $\beta$ remains little changed and IL- 6 levels where higher in both groups. This immune cascade can be attenuated by ERAS compared with conventional postoperative care [22].

Considering the reduced manipulation using minimally invasive surgical techniques, the surgical approach and its impact on immune response came increasingly into the focus of investigation. Postoperative leukocyte (LC) function is restored earlier using laparoscopic surgery [48] and laparoscopy reduces the activation of mast cells and thus postsurgical inflammation and POI [49]. A recent meta-analysis including 13 studies demonstrated that open colorectal surgery for neoplasia is associated with higher serum levels of IL-6 and IL-1, inducing an intense immune and inflammatory response compared with laparoscopy [50]. In contrast, in another meta-analysis including 11 trials, sufficient evidence to support superior preservation of global immune function (T-suppressor lymphocytes CD8+, C-reactive protein) with laparoscopy could not be reached [51]. Therefore, the potential of postsurgical inflammatory reduction by laparoscopy needs to be investigated in further studies.

Anti-inflammatory effects of dexamethasone in ERAS have been discussed in several studies. A meta-analysis including 11 RCTs showed that preoperative single-dose glucocorticoid administration decreases the rate of postsurgical complications and LOS, most likely as a consequence of attenuating the inflammatory response after major abdominal surgery [52]. Another meta-analysis emphasized the role of dexamethasone in the multimodal analgesic concept, showing that at doses of more than $0.1 \mathrm{mg} / \mathrm{kg}$ it is an effective adjunct to reduce postoperative pain and opioid consumption [53]. 
The approach of perioperative immune nutrition has been investigated in a meta-analysis including 13 RCTs. Immune nutrition (e.g. glutamine, arginine, omega-3 polyunsaturated fatty acids and ribonucleic acids) had no significant effect on postoperative mortality, but had positive effects on postoperative infection rate, LOS and improved immune function, increasing total lymphocyte counts, CD4 levels, IgG levels and decreasing IL-6 levels [54]. The role of neuropeptides in gastrointestinal physiology and immune regulation followed by surgery still needs to be clarified [55].

Therefore, reduction in surgical stress and immune response with attenuated tissue inflammation by using minimally invasive surgery, neuropeptides, mast cell inhibitors, cytokine antagonists and glucocorticoids should be investigated in further studies.

\section{New Approaches to Prevent POI}

POI is thought to be a consequence several tissue level pathways. Subsequently, four major pathways inducted complex immune cascades in the intestine leading to POI have been identified: the neurogenic, inflammatory, hormonal and pharmacological pathways [16]. At the site of surgical manipulation, intestinal macrophages produce mediators that lead to paralysis of the mucosal myocytes. The mechanism of activation of macrophages in physically untouched intestinal areas remains unclear. The immune response following manipulation during surgery involves dendrite cells, releasing a cascade of interleukins (IL-12), interferon- $\gamma$ (INF- $\gamma$ ) and T-helper type 1 cells, which activate macrophages. Recently, Engel et al. [56] described POI as a T-helper type 1 cell-mediated disease and identified T-helper type 1 cells as potential targets for disease monitoring and therapy. Therefore, prevention of $\mathrm{T}$ cell migration, inhibition of IL-12, TGF- $\beta$ and INF- $\gamma$ are potential treatment targets which have to be further investigated.

In experimental studies, neuronal stimulation with prokinetic 5-hydroxytryptamine-4-receptor (5-HT4R) agonists and dopamine receptor antagonists have been found to be potential therapeutic agents for directly ameliorating the motility disorder associated with POI [57]. Additionally, an experimental study showed that intestinal ghrelin (growth hormone release inducing) receptor agonists (TZP-101) improve large-bowel function in rats with POI following surgical manipulation, which suggests that it accelerates the upper gastrointestinal transit and shortens POI [58].

Our understanding of tissue level processes in POI has improved based on promising results from experimental studies. More higher-evidence studies are necessary to further investigate new immune modulation drugs, their interaction in the complex immune cascade and their possible implementation in clinical practice.

\section{Impact of Reduced Tissue Oxygenation}

Adequate tissue oxygenation is one of the preconditions of healing and recovery although it is no original ERAS item. The level of tissue oxygenation is closely correlated with GDT using colloids. This is generally explained by the fact that colloid fluid administration expands the intravascular volume, improves microcirculation and therefore improves tissue oxygenation compared with crystalloids [59]. Supplemental oxygen administration in the peri- and postoperative phase leads to improved outcome and decreased morbidity [60]. A recent study shows a relation between tissue oxygenation and surgical approach. Laparoscopy was associated with higher levels of tissue oxygenation compared with open surgery although pneumoperitoneum with increased intra-abdominal pressure and the commonly used Trendelenburg position can disturb alveolar ventilation and compromise oxygenation during laparoscopic surgery [61]. The analgesic modality (e.g. epidural analgesia, spinal analgesia, patient-controlled analgesia) has no impact on oxygen delivery during laparoscopic surgery [62].

Summing up, adequate tissue oxygenation can be improved by adequate colloid fluid administration and laparoscopic surgery, and should become a constant item in the ERAS concept.

New Metabolic and Hormonal Aspects to Improve

Postoperative Recovery

The surgical stress response can activate the hypothalamus-pituitary-adrenal axis, which leads to the release of glucocorticoids, causing postoperative insulin resistance [22]. Although insulin resistance is decreased by ERAS, additional perioperative intravenous glucose administration or preoperative carbohydrate drinks may be beneficial [63]. In an experimental study, corticotropin-releasing factor (CRF) was demonstrated to affect humoral stress response after surgery. CRF plays an important role in mediating the early phase of gastric ileus through activation of CRF-1 receptors, and therefore may be a potential target for medications preventing POI [64].

\section{GDT and Colloids in ERAS}

Perioperative fluid management has received increased interest in translational research. Only a few experimental studies provided first data that improved our 
understanding the cellular processes in fluid administration. The advantages of fluid restriction with avoidance of hypovolemia as outlined in the Consensus Guidelines [3] were challenged in an experimental pig model study [64] comparing restricted crystalloids versus GDT with crystalloids versus GDT with colloids, which found lower tissue oxygenation and decreased microcirculation using fluid restriction. While colloid fluid administration with GDT improved the microcirculation also in unaffected and perianastomotic colonic tissue, interestingly the type of fluid administration (crystalloid, colloid, restricted) had no effect on the hemodynamic parameters.

As already mentioned in the Consensus Guidelines, GDT (e.g. with transesophageal ultrasound monitoring or a central venous catheter) is superior to a non-protocol-based standard with respect to outcome (grade A) and should be considered on an individual basis [3]. This individual approach includes optimization of flow-related parameters within the limits of the individual cardiac capacity. Several RCTs provided evidence that GDT also improves the outcome regarding nausea, vomiting, postoperative ileus and LOS [65]. But GDT alone does not improve the microcirculation and tissue oxygenation. It must be combined with colloid fluid administration to provide its positive effect on anastomotic healing [62]. Noblett et al. [66] highlighted the effect of GDT on the postoperative immune response, showing lower IL- 6 levels in GDT and thus attenuation of stress-induced organ dysfunction.

In summary, colloids for GDT were used in several clinical and experimental studies, supporting the trend of better outcomes compared with crystalloids and providing longer intravascular microcirculation and tissue oxygenation.

New Pharmacological Aspects to Improve

Postoperative Recovery (ERAS)

Several trials failed to establish a significant reduction in POI and LOS using well-known prokinetic medications (e.g. metoclopramid, cisapride, erythromycine or prostigmine) [67]. Therefore, a critical review of conventional medical approaches, the alternative use of local anesthetics and implementation of new medications (alvimopan, capsaicin and mosapride citrate) became the objective of translational research in ERAS.

Local anesthetics (e.g. lidocain) are well known in surgery and widely used to prevent postoperative pain. Meta-analyses favored intraperitoneal administration of local anesthetics [68] and intravenous lidocain administration, with clear advantages in patients undergoing major

Fast-Track Surgery - Conditions and Challenges in Postsurgical Treatment abdominal surgery with faster return of bowel function and decreased LOS [69].

Capsaicin is a new substance, known to cause enhanced sensitivity to noxious stimuli, followed by a period with reduced sensitivity and persistent desensitization after repeated local applications. A meta-analysis based on the Cochrane database and including 6 RCTs showed that repeated application of a low-dose capsaicin cream $(0.075 \%)$ or a single application of a high-dose $(8 \%)$ patch may provide a degree of pain relief to some patients with painful neuropathic conditions [70]. Thus, its role in the multimodal pain concept improving ERAS needs to be further investigated.

Alvimopan, a selective intestinal $\mu$-receptor opioid antagonist, has been the subject of several meta-analyses to investigate its impact on postoperative recovery, attenuation of negative effects of opioids and reduction in the occurrence of POI. The United States Federal Drug Administration (US FDA) approved alvimopan (Entereg ${ }^{\circledR}$ ) in 2008 for oral intake before and after large and small bowel resection to prevent POI [71]. Although alvimopan has been shown to save overall hospital costs [72] and accelerate gastrointestinal recovery following major abdominal surgery [73], collection of further data appears to be important to reach higher levels of evidence and implementation in routine ERAS practice. This is all the more important, considering that recent data challenge its impact in laparoscopy [74] and its incorporation into ERAS practice is less than expected by the data [75].

New prokinetic medications are part of the ongoing discussion. Laxatives, e.g. magnesium oxide, did not show any beneficial effects in ERAS in a recent randomized study [76]. Mosapride citrate, a 5-HT4R, reduced the occurrence of POI in a clinical study [77].

\section{Conclusion}

ERAS and its items have been the subject of several clinical trials that obtained significant evidence for improved postoperative outcome compared with conventional postoperative care. Based on the Consensus Guidelines [3], ERAS is subject to evolution regarding the clinical implementation and modification of its items. Although ERAS significantly decreases LOS and the overall occurrence of complications, compliance with its protocol in daily clinical practice worldwide is not optimal [7]. Considering multidisciplinary in ERAS, cooperation with anesthetists, nurses, physiotherapists, out- 
patient care and general practitioners is mandatory to further improve implementation and thus the outcome and success of ERAS. This review further emphasizes that knowledge regarding underlying tissue level processes and complex immune inflammatory cascades is limited. First promising results from experimental and pharmacological studies need to translate into the clinical experimental setting with the aim of reaching higher levels of evidence.

Keeping up with the evolution of the ERAS protocol in the last decade, it may be challenging to omit individual items or replace and modify them in order to reach better outcomes. Several studies suggest that recovery after surgery may rather be influenced by the surgical approach then by the ERAS items per se, which may overreach minimally invasive techniques compared with the other ERAS items. There is no doubt that the role of epidural anesthesia especially in laparoscopy and ERAS needs to be reviewed critically due to controversial results, lack of evident data and side effects.

The humoral, immune and inflammatory response after abdominal surgery is one of the major topics in translational research. Since inflammatory processes are known to induce POI based on a cascade of mediators (IL, TNF), several tissue level treatment options have been investigated. Reduced activation of mast cells by laparoscopy, pharmacological stabilization of mast cell degranulation and central activation of cholinergic antiinflammatory pathways are promising approaches to prevent POI. The surgical access seems to have an important impact, but its clinical significance regarding immune, inflammatory and humoral response and recovery is still uncertain and needs further research. Dexamethasone can reduce complications, LOS and inflammatory response in the clinical setting. But similarly to immune modulation and immune nutrition, its role in the ERAS concept needs further clarification. New approaches to prevent POI are based on intra- and intercellular processes. Since animal studies have revealed that POI is a T-helper type-1 cell-mediated disease, prevention of T cell migration, inhibition of IL-12, TGF- $\beta$ and INF$\gamma$ is a potential treatment option, which has to be further investigated in clinical settings. Agonists of 5-HT4R, of the dopamine receptor and the ghrelin receptor may be helpful to overcome POI and present interesting targets for further investigations.

Using laparoscopy rather than open surgery increases tissue oxygenation whereas the analgesic regimen has no effect on perioperative oxygen delivery. To reduce the postoperative insulin resistance, perioperative glucose administration and preoperative carbohydrate drinks can improve the outcome and reduce LOS.

GDT facilitates faster recovery of gastrointestinal function (POI) and better microcirculation. But GDT needs to be combined with colloid fluid administration to provide its full effect. But still, there is a lack of data integrating GDT and colloid fluid administration in routine ERAS and the practicing anesthetist is still left with some unanswered questions.

New drugs have been investigated to improve the outcome in ERAS. Alvimopan is one of the new medications and has been increasingly used in clinical studies. It can reduce LOS and the occurrence of POI, but its clinical implementation into the ERAS concept is less then expected. Since the US FDA approved alvimopan (Entereg) in 2008, further clinical studies investigating the impact of alvimopan in the ERAS concept are needed. Capsaicin is only beneficial in local administration in chronic pain, which challenges its role in ERAS. Local anesthetics provide evidence-improving data in ERAS, considering its known use in local and regional anesthesia (e.g. TAP block), but also showed promising effects in reducing POI and LOS when administered intravenously.

In summary, the modalities of the ERAS protocol are subject to a constant evolution. It may be difficult significantly to improve the good results of the ERAS protocol by adding further items. Translational research topics may have higher potential for improving outcomes in ERAS. But considering the limited knowledge in basic research topics, further investigation of tissue level processes is mandatory.

\section{Disclosure Statement}

The authors have no ethical problems or conflicts of interests to declare; there are no financial and personal relationships with other people or organizations that could have inappropriately influenced this article.

References

$\checkmark 1$ Kehlet H: Multimodal approach to control postoperative pathophysiology and rehabilitation. Br J Anaest 1997;78:606-617.

2 Kehlet H: Fast-track colonic surgery: status and perspectives. Recent Results Cancer Res $2005 ; 165: 8-13$

-3 Lassen K, Soop M, Nygren J, Cox PBW, Hendry PO, Spies C: Consensus review of optimal perioperative care in colorectal surgery: Enhanced Recovery After Surgery (ERAS) Group recommendations. Arch Surg 2009; 144:961-969. 
4 Vlug MS, Wind J, Hollmann MW, Ubbink DT, Cense HA, Engel AF: Laparoscopy in combination with fast track multimodal management is the best perioperative strategy in patients undergoing colonic surgery: a randomized clinical trial (LAFA study). Ann Surg 2011;254:868-875.

$\checkmark 5$ Varadhan KK, Neal KR, Dejong CHC, Fearon $\mathrm{KCH}$, Ljungqvist O, Lobo DN: The enhanced recovery after surgery (ERAS) pathway for patients undergoing major elective open colorectal surgery: a meta-analysis of randomized controlled trials. Clin Nutr 2010;29:434-440.

6 Spanjersberg WR, Reurings J, Keus F, van Laarhoven CJ: Fast track surgery versus conventional recovery strategies for colorectal surgery. Cochrane Database Syst Rev 2011;CD007635.

7 Ahmed J, Khan S, Lim M, Chandrasekeran T, Macfie J: Enhanced recovery after surgery protocols - Compliance and variations in practice during routine colorectal surgery. Colorectal Dis DOI: 10.1111/j.14631318.2011.02856.

${ }_{8}$ Ramírez JM, Blasco JA, Roig JV, MaesoMartínez S, Casal JE, Esteban F: Enhanced recovery in colorectal surgery: a multicentre study. BMC Surg 2011;11:9.

$>9$ Hasenberg T, Keese M, Längle F, Reibenwein B, Schindler K, Herold A. 'Fast-track' colonic surgery in Austria and Germany - results from the survey on patterns in current perioperative practice. Colorectal Dis 2009;11: 162-167.

10 Hoffmann H, Kettelhack C: Fast-TrackChirurgie - Voraussetzungen und Herausforderungen in der Nachbehandlung. Ther Umsch 2012;69(1):9-13.

$\checkmark 11$ Maessen J, Dejong CHC, Hausel J, Nygren J, Lassen K, Andersen J: A protocol is not enough to implement an enhanced recovery programme for colorectal resection. Br J Surg 2007;94:224-231.

12 Gustafsson UO, Hausel J, Thorell A, Ljungqvist O, Soop M, Nygren J: Adherence to the enhanced recovery after surgery protocol and outcomes after colorectal cancer surgery. Arch Surg 2011;146:571-577.

-13 Nygren J, Hausel J, Kehlet H, Revhaug A, Lassen K, Dejong C: A comparison in five European centres of case mix, clinical management and outcomes following either conventional or fast-track perioperative care in colorectal surgery. Clin Nutr 2005;24:455461.

14 Schwenk W, Günther N, Wendling P, Schmid M, Probst W, Kipfmüller K: 'Fast-track' rehabilitation for elective colonic surgery in Germany - prospective observational data from a multi-centre quality assurance programme. Int J Colorectal Dis 2008;23:93-99.

-15 Kahokehr A, Sammour T, Zargar-Shoshtari K, Thompson L, Hill AG: Implementation of ERAS and how to overcome the barriers. Int J Surg 2009;7:16-19.
16 Luckey A, Livingston E, Taché Y: Mechanisms and treatment of postoperative ileus. Arch Surg 2003;138:206-214.

17 Iyer S, Saunders WB, Stemkowski S: Economic burden of postoperative ileus associated with colectomy in the United States. J Manag Care Pharm 2009;15:485-494.

18 Delaney CP, Marcello PW, Sonoda T, Wise P, Bauer J, Techner L: Gastrointestinal recovery after laparoscopic colectomy: results of a prospective, observational, multicenter study. Surg Endosc 2010;24:653-661.

19 Augestad KM, Delaney CP: Postoperative ileus: impact of pharmacological treatment, laparoscopic surgery and enhanced recovery pathways. World J Gastroenterol 2010;16: 2067-2074.

20 Scabini S, Rimini E, Romairone E, Scordamaglia R, Damiani G, Pertile D: Colon and rectal surgery for cancer without mechanical bowel preparation: one-center randomized prospective trial. World J Surg Oncol 2010;8:35.

21 Delaney CP, Chang E, Senagore AJ, Broder M: Clinical outcomes and resource utilization associated with laparoscopic and open colectomy using a large national database. Ann Surg 2008;247:819-824.

22 Ren L, Zhu D, Wei Y, Pan X, Liang L, Xu J: Enhanced Recovery After Surgery (ERAS) Program attenuates stress and accelerates recovery in patients after radical resection for colorectal cancer: a prospective randomized controlled trial. World J Surg 2012;36:407414.

23 Adamina M, Kehlet H, Tomlinson GA, Senagore AJ, Delaney CP: Enhanced recovery pathways optimize health outcomes and resource utilization: a meta-analysis of randomized controlled trials in colorectal surgery. Surgery 2011;149:830-840.

24 Vlug MS, Bartels SAL, Wind J, Ubbink DT, Hollmann MW, Bemelman WA. Which fast track elements predict early recovery after colon cancer surgery? Colorectal Dis DOI: 10.1111/j.1463-1318.2011.02854

25 Barletta JF, Asgeirsson T, Senagore AJ: Influence of intravenous opioid dose on postoperative ileus. Ann Pharmacother 2011;45: 916-923.

26 Marret E, Remy C, Bonnet F: Meta-analysis of epidural analgesia versus parenteral opioid analgesia after colorectal surgery. $\mathrm{Br} \mathrm{J}$ Surg 2007;94:665-673.

-27 Turunen P, Carpelan-Holmström M, Kairaluoma $\mathrm{P}$, Wikström $\mathrm{H}$, Kruuna $\mathrm{O}$, Pere $\mathrm{P}$ : Epidural analgesia diminished pain but did not otherwise improve enhanced recovery after laparoscopic sigmoidectomy: a prospective randomized study. Surg Endosc 2009;23:31-37.

28 Braga M, Vignali A, Zuliani W, Frasson M, Di Serio C, Di Carlo V: Laparoscopic versus open colorectal surgery. Ann Surg 2005;242: 890-896.
29 Delaney CP, Kiran RP, Senagore AJ, Brady K, Fazio VW: Case-matched comparison of clinical and financial outcome after laparoscopic or open colorectal surgery. Ann Surg 2003;238:67-72.

30 Nelson R, Edwards S, Tse B: Prophylactic nasogastric decompression after abdominal surgery. Cochrane Database Syst Rev 2007;CD004929.

- 31 van Bree S, Vlug M, Bemelman W, Hollmann M, Ubbink D, Zwinderman K: Faster recovery of gastrointestinal transit after laparoscopy and fast-track care in patients undergoing colonic surgery. Gastroenterology 2011;141:872-880.

32 White PF, Kehlet H, Neal JM, Schricker T, Carr DB, Carli F: The role of the anesthesiologist in fast-track surgery: from multimodal analgesia to perioperative medical care. Anesth Analg 2007;104:1380-1396, table of contents.

33 Carli F, Baldini G: Fast-track surgery: it is time for the anesthesiologist to get involved! Minerva Anestesiol 2011;77:227-230.

34 Jørgensen H, Wetterslev J, Møiniche S, Dahl JB: Epidural local anaesthetics versus opioidbased analgesic regimens on postoperative gastrointestinal paralysis, PONV and pain after abdominal surgery. Cochrane Database Syst Rev 2000;CD001893.

35 Low J, Johnston N, Morris C: Epidural analgesia: first do no harm. Anaesthesia 2008;63:1-3.

36 Levy BF, Fawcett WJ, Scott MJP, Rockall TA. Intra-operative oxygen delivery in infusion volume optimized patients undergoing laparoscopic colorectal surgery within an enhanced recovery programme: the effect of different analgesic modalities. Colorectal Dis DOI: $10.1111 / \mathrm{j} .1463-1318.2011 .02805$.

37 Kehlet H: Epidural analgesia and recovery after abdominal surgery. Am J Surg 2007; 193:291.

38 Carli F, Kehlet H, Baldini G, Steel A, McRae $\mathrm{K}$, Slinger P: Evidence basis for regional anesthesia in multidisciplinary fast-track surgical care pathways. Reg Anesth Pain Med 2011;36:63-72.

39 Kehlet H: Epidural analgesia and postoperative outcome. Lancet 2008;372:2109;author reply $2110-2111$.

40 Levy BF, Tilney HS, Dowson HMP, Rockall TA: A systematic review of postoperative analgesia following laparoscopic colorectal surgery. Colorectal Dis 2010;12:5-15.

41 Sim R, Cheong DM, Wong KS, Lee BMK, Liew QY: Prospective randomized, doubleblind, placebo-controlled study of pre- and postoperative administration of a COX-2specific inhibitor as opioid-sparing analgesia in major colorectal surgery. Colorectal Dis 2007;9:52-60.

42 Eskicioglu C, Forbes SS, Aarts MA, Okrainec A, McLeod RS: Enhanced recovery after surgery (ERAS) programs for patients having colorectal surgery: a meta-analysis of randomized trials. J Gastrointest Surg 2009;13: 2321-2329. 
-43 Gouvas N, Tan E, Windsor A, Xynos E, Tekkis PP: Fast-track vs standard care in colorectal surgery: a meta-analysis update. Int J Colorectal Dis 2009;24:1119-1131.

$\checkmark 44$ Boeckxstaens GE: Neuroimmune interaction in the gut: from bench to bedside. Verh K Acad Geneeskd Belg 2006;68:329-355.

- 45 De Winter BY, van den Wijngaard RM,de Jonge WJ: Intestinal mast cells in gut inflammation and motility disturbances. Biochim Biophys Acta 2012;1822:66-73.

-46 Boeckxstaens GE, de Jonge WJ: Neuroimmune mechanisms in postoperative ileus. Gut 2009;58:1300-1311.

47 The F, Cailotto C, van der Vliet J, de Jonge WJ, Bennink RJ, Buijs RM: Central activation of the cholinergic anti-inflammatory pathway reduces surgical inflammation in experimental post-operative ileus. Br J Pharmacol 2011;163:1007-1016.

-48 Braga M, Vignali A, Gianotti L, Zuliani W, Radaelli G, Gruarin P, et al: Laparoscopic versus open colorectal surgery: a randomized trial on short-term outcome. Ann Surg 2002;236:759-766; discussion 767.

-49 The FO, Buist MR, Lei A, Bennink RJ, Hofland J, van den Wijngaard RM: The role of mast cell stabilization in treatment of postoperative ileus: a pilot study. Am J Gastroenterol 2009; 104:2257-2266.

-50 Sammour T, Kahokehr A, Zargar-Shoshtari K, Hill AG: A Prospective case-control study of the local and systemic cytokine response after laparoscopic versus open colonic surgery. J Surg Res 2012;173:278-285.

51 Liu C, Liu J, Zhang S: Laparoscopic versus conventional open surgery for immune function in patients with colorectal cancer. Int J Colorectal Dis 2011;26:1375-1385.

-52 Srinivasa S, Kahokehr AA, Yu T-C, Hill AG: Preoperative glucocorticoid use in major abdominal surgery: systematic review and meta-analysis of randomized trials. Ann Surg 2011;254:183-191.

-53 De Oliveira GS, Almeida MD, Benzon HT, McCarthy RJ: Perioperative single dose systemic dexamethasone for postoperative pain: a meta-analysis of randomized controlled trials. Anesthesiology 2011;115:575588.

54 Zheng Y, Li F, Qi B, Luo B, Sun H, Liu S: Application of perioperative immunonutrition for gastrointestinal surgery: a meta-analysis of randomized controlled trials. Asia Pac J Clin Nutr 2007;16(suppl 1):253-257. de Jonge WJ, Greaves DR: Immune modulation in gastrointestinal disorders: new opportunities for therapeutic peptides? Expert Rev Gastroenterol Hepatol 2008;2:741-748.

56 Engel DR, Koscielny A, Wehner S, Maurer J, Schiwon M, Franken L: T helper type 1 memory cells disseminate postoperative ileus over the entire intestinal tract. Nat Med 2010;16:1407-1413.

57 Tsuchida Y, Hatao F, Fujisawa M, Murata T, Kaminishi M, Seto Y: Neuronal stimulation with 5-hydroxytryptamine 4 receptor induces anti-inflammatory actions via $\alpha 7 \mathrm{n}$ ACh receptors on muscularis macrophages associated with postoperative ileus. Gut 2011;60:638-647.

58 Fraser GL, Venkova K, Hoveyda HR, Thomas $\mathrm{H}$, Greenwood-Van Meerveld B: Effect of the ghrelin receptor agonist TZP-101 on colonic transit in a rat model of postoperative ileus. Eur J Pharmacol 2009;604:132-137.

59 Lang K, Boldt J, Suttner S, Haisch G: Colloids versus crystalloids and tissue oxygen tension in patients undergoing major abdominal surgery. Anesth Analg 2001;93:405-409.

60 Belda FJ, Aguilera L, García de la Asunción J, Alberti J, Vicente R, Ferrándiz L: Supplemental perioperative oxygen and the risk of surgical wound infection: a randomized controlled trial. JAMA 2005;294:2035-2042.

61 Crozier TA: Anesthesiologic aspects of minimally invasive surgery. Zentralbl Chir 1993; 118:573-581.

62 Kimberger O, Arnberger M, Brandt S, Plock J, Sigurdsson GH, Kurz A: Goal-directed colloid administration improves the microcirculation of healthy and perianastomotic colon. Anesthesiology 2009;110:496-504.

63 Ljungqvist O, Nygren J, Thorell A: Insulin resistance and elective surgery. Surgery 2000;128:757-760.

64 Luckey A, Wang L, Jamieson PM, Basa NR, Million M, Czimmer J: Corticotropin-releasing factor receptor 1-deficient mice do not develop postoperative gastric ileus. Gastroenterology 2003;125:654-659.

65 Bundgaard-Nielsen M, Holte K, Secher NH, Kehlet $\mathrm{H}$ : Monitoring of peri-operative fluid administration by individualized goal-directed therapy. Acta Anaesthesiol Scand 2007;51:331-340.

66 Noblett SE, Snowden CP, Shenton BK, Horgan AF: Randomized clinical trial assessing the effect of Doppler-optimized fluid management on outcome after elective colorectal resection.Br J Surg 2006;93:1069-1076.
67 Bungard TJ, Kale-Pradhan PB: Prokinetic agents for the treatment of postoperative ileus in adults: a review of the literature. Pharmacotherapy 1999;19:416-423.

68 Kahokehr A, Sammour T, Soop M, Hill AG: Intraperitoneal use of local anesthetic in laparoscopic cholecystectomy: systematic review and metaanalysis of randomized controlled trials. J Hepatobiliary Pancreat Sci 2010;17:637-656.

69 McCarthy GC, Megalla SA, Habib AS: Impact of intravenous lidocaine infusion on postoperative analgesia and recovery from surgery: a systematic review of randomized controlled trials. Drugs 2010;70:1149-1163.

70 Derry S, Lloyd R, Moore RA, McQuay HJ: Topical capsaicin for chronic neuropathic pain in adults. Cochrane Database Syst Rev 2009;CD007393.

71 Becker G, Blum HE: Novel opioid antagonists for opioid-induced bowel dysfunction and postoperative ileus. Lancet 2009;373: 1198-1206.

72 Bell TJ, Poston SA, Kraft MD, Senagore AJ, Delaney CP, Techner L: Economic analysis of alvimopan in North American phase III efficacy trials. Am J Health Syst Pharm 2009; 66:1362-1368

73 Tan EK, Cornish J, Darzi AW, Tekkis PP: Meta-analysis: alvimopan vs. placebo in the treatment of post-operative ileus. Aliment Pharmacol Ther 2007;25:47-57.

-74 Ludwig K, Enker WE, Delaney CP, Wolff BG, Du W, Fort JG: Gastrointestinal tract recovery in patients undergoing bowel resection: results of a randomized trial of alvimopan and placebo with a standardized accelerated postoperative care pathway. Arch Surg 2008; 143:1098-1105.

75 Abodeely A, Schechter S, Klipfel A, Vrees M, Lagares-Garcia J: Does alvimopan enhance return of bowel function in laparoscopic right colectomy? Am Surg 2011;77:14601462.

76 Andersen J, Christensen H, Pachler JH, Hallin M, Thaysen HV, Kehlet H. Effect of the laxative magnesium oxide on gastrointestinal functional recovery in fast-track colonic resection: a double-blind, placebo-controlled randomized study. Colorectal Dis 2011;14:776-782

77 Toyomasu Y, Mochiki E, Morita H, Ogawa A, Yanai M, Ohno T: Mosapride citrate improves postoperative ileus of patients with colectomy. J Gastrointest Surg 2011;15:13611367. 